\title{
On the 25th Anniversary of the ADA: How Inclusive Are Campus Recreation Programs?
}

\author{
Sarah J. Young, William D. Ramos, \\ Sherril L. York, and Allison L. Fletcher
}

\begin{abstract}
On the 25th anniversary of the Americans with Disabilities Act, it is well known that postsecondary institutions' programs, including campus recreation, must be accessible to people with disabilities. This study critiqued the inclusiveness, and specifically, the welcoming environment of campus recreation programs in the Big Ten by conducting a content analysis of those institutions' websites. Campus recreation programs were targeted because their purpose is to serve the recreational sport needs of all students, faculty, and staff in the campus community. The content analysis used enumerative techniques for determining frequencies as well as qualitative coding techniques of manifest and latent coding. Enumerative results indicated the majority of schools offered minimal programming and equipment for people with disabilities while four themes emerged from the qualitative analysis. The results are discussed along with recommendations for campus recreation programs in creating a welcoming environment and a more inclusive culture for students with disabilities.
\end{abstract}

The year 2015 marked the 25th anniversary of the Americans with Disability Act (ADA). Enacted into law in 1990, the ADA is one of the most comprehensive pieces of civil rights legislation prohibiting discrimination against individuals with disabilities. The ADA guarantees that people with disabilities have the same rights and opportunities as everyone else to participate in all areas of public life, including jobs, schools, transportation, and all public and private settings open to accommodate the general public. Following the lead of the Civil Rights Act of 1964, which prohibits discrimination on the basis of race, color, religion, sex, or national origin and Section 504 of the Rehabilitation Act of 1973, "the ADA is an equal opportunity law for people with disabilities" (United States Department of Justice (b), n.d.).

Campus recreational sport has existed since 1913 with the first recognized intramural sports programs at Ohio State University and University of Michigan (Ohio State Recreational Sports, 2015; University of Michigan, 2015). The purpose of recreational sport is the "delivery of sport for the sake of fitness and fun" (Mull, Forrester, \& Barnes, 2013, p. 11). On college campuses, the sport skill level of the

Young (sarjyoun@indiana.edu), Ramos, and Fletcher are with Indiana University. York is with the National Center on Accessibility. 
majority of students is average to below average, making campus recreation programs the perfect fit to meet most students' sport participation needs and desires. The National Intramural-Recreational Sports Association (NIRSA) a professional organization supporting collegiate recreation promotes the values of diversity and inclusion on college campuses (NIRSA (a), n.d.), thus the philosophy of campus recreation programs parallels that of the ADA by providing opportunities for all students. Yet, how well students with disabilities are being included in these programs varies.

Shortly following the enactment of the ADA, Roth and Hudson (1994) explained the mandates of the law as well as how the ADA would impact campus recreational sport programs. While it was acknowledged that budgets, programs, planning processes, and facilities would all be impacted, their primary point was the obligation of recreational sport providers to make necessary changes and "allow all students the opportunity to focus on their abilities, not their disabilities" (p. 24). Green and De Coux (1996) concurred by discussing how students with disabilities could be better included in campus recreational sport programs. They also provided a discussion of future trends and recommendations that would likely impact the development and growth of campus recreational sport programs integrating students with disabilities. The foundation for compliance with the ADA was established early on. The popularity and benefits of campus recreation for a majority of students is well documented and has been sustained for over a century of student participation (Forrester, 2015; Kerr-Downs Research, 2003; NIRSA/NASPA, 2010). But, since the enactment of the ADA, do students with disabilities find campus recreation services as inviting as students without disabilities?

Postsecondary educational institutions are covered under Titles II and III of the ADA and must insure that the programs offered, including campus recreational sport, are accessible to students with disabilities (Leuchovius, 2003). This was most recently reinforced by the U.S. Department of Education in its January 2013 Dear Colleague Letter providing guidance on the obligations of public schools to provide an equal opportunity to participate in extracurricular activities. Of note in the guidance is "students at the postsecondary level must also be provided an equal opportunity to participate in athletics, including intercollegiate, club, and intramural athletics" (U.S. Department of Justice, 2013, p. 1). In a search of case law, no specific cases claiming discrimination under the ADA by campus recreation programs against students with disabilities were found, but the question remained, do students with disabilities feel welcomed and included in the programs provided by this campus service?

A primary component of accessibility and inclusion is a welcoming environment. While not a legal requirement of the ADA, a welcoming environment expresses an underlying attitude toward individuals with disabilities characterized by accessibility statements, use of people-first language, access to the physical spaces, providing staff support, and encompassing a wide scope of program offerings. The question that became the focus of this inquiry was how well are campus recreation programs meeting the needs of students with disabilities by presenting a welcoming environment? Because a review of the literature revealed only two studies involving ADA issues and campus recreation that were conducted more than nine years ago (Fujii \& Woodard, 2006; Gillespie, 2002), this question seemed worth pursuing with an exploratory, baseline investigation. Therefore, the purpose of this article is 
to present the findings of a pilot study, which critiqued the welcoming environment presented by campus recreation programs in the Big Ten.

\section{Brief Historical Background}

The roots of the ADA stretch back to the beginning of the 20th century where concern for equal rights for underrepresented groups evolved into significant legislation relating to equal access, including rights to educational and recreational services. During the early part of this period legislation aimed toward people with disabilities focused on newly disabled veterans returning from World War I to assist them in returning to employment. Vocational rehabilitation programs were expanded to include all Americans with physical disabilities and then subsequent amendments expanded services to veterans after World War II, the Korean War, and the Vietnam War (Dieser \& Scholl, 2010).

The Architectural Barriers Act (ABA) of 1968, PL 90-480 was one of the first efforts to ensure that the built environment was accessible to people with disabilities (U.S. Access Board (a), n. d.). The ABA required that facilities designed, built or altered with federal monies or leased by federal agencies must provide physical access to people with disabilities. Nonfederal government facilities receiving federal funding were also covered by the ABA including many park and recreation departments, state departments of natural resources, state parks, and postsecondary institutions.

Up until the late 1960s, the disability rights movement floundered as disabilityrelated organizations did not speak with one voice and often pursued different goals (Stroman, 2003). In the 1970s the focus changed and people with disabilities gained more of a consensus. "Nothing about us, without us' was one of the compelling slogans to come out of the disability rights movement" (Pelka, 2012, p. ix). In 1973 the Rehabilitation Act, PL 93-112 was passed to remove architectural, employment and transportation barriers to provide equal access for people with disabilities and served as the forerunner of the Americans with Disabilities Act.

During the 1980s the movement toward greater community and travel access took place promoting more independent living for people with disabilities. Significant legislation and events included the Air Carrier Access Act, the TechnologyRelated Assistance Act for Individuals with Disabilities, and the establishment of the Center for Universal Design. Development and availability of technology devices reduced both attitudinal and physical barriers, opening up opportunities in all aspects of parks, recreation and tourism (Dieser \& Scholl, 2010).

The ADA, signed into law in 1990 by President George H. W. Bush, is the most sweeping disability rights legislation in American history creating changes in the law and strengthening the rights of people with disabilities in society (Ross \& Phillips, 1995). The Department of Justice published the Title III regulations for the ADA in July 1991, which included the 1991 ADA Standards for Accessible Design (1991 Standards) (U.S. Department of Justice (a), n. d.). The ADA Amendments Act of 2008 revised the definition of disability to more broadly cover impairments that limit a major life activity and make it easier to seek protection as well as establish that an individual has a disability under the ADA (EEO21, n. d.; U.S. Access Board (b), n. d.). In addition in September 2010, the 2010 ADA Standards for Accessible Design (2010 Standards) were published by the Department of Justice and included 
for the first time standards for recreation facilities. On March 15, 2012, compliance with the 2010 Standards was required for new construction and alterations under Titles II and III, as well as the compliance date for using the 2010 Standards for program accessibility and barrier removal.

A significant new component of the 2010 ADA regulations was the provision referred to as "safe harbor" that allows for physical elements in facilities built before March 15, 2012 to remain in place until they are altered if they complied with the 1991 ADA Standards. For example, reach ranges changed from a minimum of 9 inches and a maximum of 54 inches in the 1991 Standards to 15 inches minimum and 48 inches maximum (U.S. Department of Justice, 2010). If a facility had all its light switches installed at 54 inches, it would be safe or exempt from making any of these new changes until the facility was altered or remodeled since it complied with the 1991 Standards.

Similarly, there are elements in the 2010 Standards that were not in the 1991 Standards that are not subject to the safe harbor exemption. Among those elements that might apply to a campus setting are recreational facilities such as exercise machines and equipment, golf facilities, sports facilities, saunas and steam rooms and swimming pools. The campus recreation entity would be required to determine if readily achievable barrier removal would be required (Title III), or if the program access requirement for Title II entities would trigger a need for a structural alteration (York, 2013). The 2010 Standards require independent means of entry and exit from swimming pools. Since there were no requirements for pool entry/exit in the 1991 Standards, an entity is required to provide one or two means of entry/ exit depending on the size of the pool. Campus recreational sports programs should have completed a review of their recreational facilities for compliance with the 2010 Standards and either come into compliance by March 15, 2012 or developed a plan for achieving compliance.

\section{A Welcoming and Inclusive Environment}

It has been said that "the environment we create communicates our beliefs about the people we serve" (National Center on Domestic Violence, Trauma, \& Mental Health, 2011). Students should feel welcomed and inspired to participate in a campus recreational sports program from the moment they walk through the door. In today's world the front "door" potential participants enter is most likely through websites and marketing materials about campus recreational programs and the opportunities they afford.

The ADA requires that programs and services be provided in an integrated setting and thus inclusion of people with disabilities in recreational activities and sports is about providing the same choices and opportunities that other people have (Anderson \& Kress, 2003; Hironaka-Juteau \& Crawford, 2010). Diane Richler, President of Inclusion International stated that "inclusion is NOT a strategy to help people fit into the systems and structures which exist in our societies; it is about transforming those systems and structures to make it better for everyone" (National Center on Health, Physical Activity and Disability, n.d.). Thus true inclusion cannot be attained without system change.

There are a number of barriers to providing inclusive recreational sports activities that must be acknowledged. Typical barriers include structural, social 
and attitudinal, psychological, communication, and programmatic accommodations (Hironaka-Juteau \& Crawford, 2010). Steps toward removing barriers to inclusion is providing a welcoming environment that includes welcoming language encouraging recreation for people of all abilities, accessible recreation facilities and areas, ways to request and receive accommodations or needed support, and staff whom can work with participants with disabilities to adapt, adjust and support their needs (Anderson \& Kress, 2003; Hironaka-Juteau \& Crawford, 2010). How well campus recreational sports programs open the virtual door to demonstrate a welcoming and inclusive environment was the focus of the review of each institution's website information.

\section{Methods}

In an attempt to quantify the issue of a welcoming environment, a content analysis was performed to investigate how institutions of higher education are currently addressing the need for students with disabilities to access resources. Websites and social media have been found to be the most popular marketing tools used by campus recreation programs as well as methods used by students to access information about campus recreation programs (Kaltenbaugh, Molnar, Bonadio, Divito, $\&$ Roeder, 2011). Based on this finding, the 14 institutions known as the Big Ten were used to examine what a student interested in options for campus recreation would encounter upon making an initial inquiry through the schools' websites. Schools in the Big Ten include: (a) University of Illinois-Champaign/Urbana, (b) Indiana University, (c) University of Iowa, (d) University of Maryland, (e) University of Michigan, (f) Michigan State University, (g) University of Minnesota, (h) University of Nebraska-Lincoln, (i) Northwestern University, (j) The Ohio State University, (k) Pennsylvania State University, (l) Purdue University, (m) Rutgers University-New Brunswick, (n) University of Wisconsin-Madison. The study was conducted from the primary investigators home institution in July and August of 2015, and was exempt from approval by the institutional review board due to the fact that no contact was made with human subjects during the study.

Content analysis is a research technique for "making replicable and valid inferences from text (or other meaningful matter) to the contexts of their use" (Krippendorff, 2013, p. 24). Weber (1990) asserted that the "central idea in analysis is that the many words of the text are classified into much fewer content categories" (p. 12). Since the current study involved the review of main university websites as well as the campus recreation website of each Big Ten university, content analysis was chosen as the most appropriate method of achieving a meaningful outcome. In establishing the framework of a content analysis, data collection units and units of analysis must be defined (White \& Marsh, 2006). The data collection units for this study were the websites of each Big Ten university's campus recreation program as well as the institutions disability student services site. Berg and Lune (2012) suggested a blended approach of content analysis utilizing qualitative methods. Units of analysis defined for measurement using manifest coding, included programming and equipment while latent coded items were used to establish emergent themes and obtain a global view of the data (Neuman, 2004).

To review student access to information about available services with regards to disabilities, each school's website was viewed from the perspective of a first attempt 
to locate relevant information. The research team determined this perspective to be a valid point of entry since searching for information on the internet is the most likely means by which today's college student would make initial contact with an institution or would find information about campus services (Fujii \& Woodard, 2006; Kaltenbaugh et al., 2011). Although it is understood that additional and more invasive methods of data collection could also be employed, it was determined that using information based on the purity of an initial entry experience was valid and representative of a true user experience and worthy of investigation on its own for this study.

Training for the coders involved reviewing research goals, established guidelines and definitions to be used for recording items in the coding schematic. For greater reliability, it was recommended by the literature to use two data coders to review the content of the websites (Henderson \& Bialeschki, 2002). The two members of the research team designated as coders performed an initial review of one chosen site, which was performed independently then discussed. This process led to discussions that allowed for questions to be asked back to the research team for clarification and confirmation before proceeding with the remainder of the coding.

The analysis began by searching a school's office of disability student services site then progressing to their campus recreation website. Tallies were kept for each item within programming and equipment categories with notes kept by researchers for wording relevant to themes observed during coding. Each of two researchers coded the data and then merged their information for analysis, discussion, and agreement. Within the categories using manifest coding it was necessary to have agreed upon definitions for what would and would not be included for programming and equipment. For example in the programming category, only those items demonstrating programs specifically designed for a person with a disability were counted. Not included were programs available to all students, faculty, and staff, which indicated possible inclusion upon request. For equipment, researchers tabulated items specifically designed to be used by participants with disabilities to engage in a recreational activity that were either mentioned or visible in website marketing. These did not include items required by the 2010 Standards for access to facilities such as aquatic pool lifts. Finally, traditional equipment that could be used without specific adaptation was also not counted. For the categories assessed using a counting approach, data were entered into the software application Statistical Package for the Social Sciences (SPSS) and was used to perform a Cohen's kappa analysis to determine intercoder reliability. Cohen's kappa was determined to be an acceptable mean by which to gauge intercoder reliability based on agreement with the five assumptions by which it's governed (Landis \& Koch, 1977). It is well established in the literature that an acceptable rate for intercoder reliability is as follows in regards to agreement: 1) $0.0-0.2=$ slight; 2) $0.21-.40=$ fair; 3 ) $0.41-0.60=$ moderate; 4) $0.61-0.80=$ substantial; and 5) $0.81-1.00=$ almost perfect (Landis \& Koch, 1977).

For the development of overall themes, members of the research team independently coded the data compiling information regarding messages being created by website content. The investigation excluded the need to make note of content related to programming and equipment since that information was gathered using a manifest type coding system. Researchers reviewed the websites several times to establish reliability in their notations then independently formed emergent themes. 
In a final step to establish the level of validity within the data, researchers met to compare, discuss, and come to agreement on final emergent themes.

\section{Results}

Each of the 14 Big Ten school websites was analyzed for the mention of programming and equipment through enumeration which was determined to be the best analysis technique for dealing with frequencies (Potter, 1996). Whenever multiple coders are used in content analysis, a check for consistency must always be made (Neuman, 2004). An intercoder reliability coefficient was calculated using a Kappa analysis and indicated $0.876(p<.0 .001), 95 \%$ CI $(0.640,1.111)$ which was determined to be substantial and acceptable to proceed with interpretation. Among the 14 schools, the coders counted an average of 13 total programs specific for students with disabilities. A mean score was used because there were differences between the two coders. The highest number of programs for any institution was four offered by one university while eight others offered 1-2 programs each. Five universities indicated no noticeable disability-specific programming. What was learned from the coding process was that for many of the schools their website information was delegated to restating existing programs for the mass student body with a mention for students looking for adaptation to contact campus recreation for further discussion. Furthermore, two schools directed students to an external program offered through academic departments which was not perceived as truly inclusive.

Overall, equipment numbers were very low meaning most of the schools reviewed in this study did not show evidence of adaptive equipment for students with disabilities on their website. The intercoder reliability coefficient for equipment was calculated at $0.736(p<.001), 95 \% \mathrm{CI}(0.406,1.06)$. Once again the results indicated that the level of agreement was acceptable to proceed with interpretation. The average count between the two coders for equipment was 11 total pieces of adaptive equipment for students with disabilities among all 14 schools with a range from 0 to 5 at any one institution. Eight of the 14 schools recorded a score of zero in this category.

In terms of emergent themes, agreement between the coders was made on several themes which clearly emerged from the data. The first theme related to the ease and fluidity that was needed to navigate the website to find information about program options for students with disabilities to participate in campus recreation. In most cases there was little to no direct connection between a school's disability student services website and the campus recreation website. Only two of the 14 institutions demonstrated a direct link between these two campus student services.

A second theme was the use of language. It was discovered that those schools providing information on program options for students, faculty, and staff with disabilities in campus recreation used language that was both positive and appropriate. For those programs in which people with disabilities were specifically addressed, people first language was most frequently used. The majority of schools had language in their mission statements that provided a sense of inclusion with the most commonly cited term being "community" which was used to infer that everyone was welcome.

A third theme identified was access to facilities which revealed that it was more common for schools to provide information on the accessibility of facilities than to 
provide information about program options available for active participation. This theme was supported by language regarding amenities such as parking, entrances, ramps, and swimming pool lifts. Although this was deemed important information the researchers did not perceive these amenities to reflect full inclusion efforts if no other specific programming information was provided. Within campus recreation websites, when available, a link to more information for people with disabilities was moderately easy to find. Only two institutions had a link that was prominent on the campus recreation main website page. It was more common, when present, for the link to be at a second or third tier level often located in a lighter font at the very bottom of the page as part of the site summary. In addition, relevant information could sometimes be found under the title of "facilities" which was not the most intuitive and added support to the earlier theme that the emphasis was on access and not as much on programming.

The fourth and final theme was access to accommodation and inclusion support. Contact information was often provided but was very general in nature to member services representatives. Although there were two examples of direct connections for support services it was not the norm for the majority of schools reviewed.

\section{Discussion}

The fundamental purpose of the ADA is integration of people with disabilities into the mainstream of American life (U.S. Department of Justice, 2015). Therefore, the ADA requires that programs and services must be provided in an integrated setting. Often, the first introduction to if and how a campus recreational sports program provides equal opportunities to all abilities is through its website information. This perspective was also shared by Fujii and Woodard (2006) who conducted a study of accessibility of campus recreation programs for students with disabilities.

Where accessibility and inclusion information was located on a website, how specific that information was, how easy it was to find and navigate, as well as cross-posting of information or links between the university's disability student services websites emerged in the first theme of the findings. Most of the schools had general statements of inclusion of all students or valuing diversity but not specific, highly placed information on accessibility or requesting services for students with disabilities. This required navigating to multiple lower levels of the site to find information or referral to a phone number for further assistance. Only two universities had cross connections between campus recreation and disability student services. As a result, the more prominent and easy the program inclusion and accessibility information is to find on a campus recreational sports website, not only is it more effective for its intended audience, but the more a welcoming environment is communicated.

The use of positive and inclusive language also contributes to a welcoming environment. In theme two, use of language, most schools illustrated appropriate use of people first language when referring to people with disabilities. As previously mentioned, many schools described their programs as inclusive of everyone, but did not have specific information about how individuals with disabilities would be included in programs. Fujii and Woodard (2006) discovered a similar result in that while campus recreation programs were made available to the entire campus community, no information was offered as to how these programs 
could be accessed and programs specifically for individuals with disabilities were the least offered.

Theme three related to the prevalence of information about the physical accessibility of the campus recreational sports facilities. It was not surprising that far more information was available about the accessible features of the physical environment such as pool lifts, accessible parking and entrances since the ADA standards for the physical environment are much more specific and measurable than the program requirements of the ADA. Yet, as Fujii and Woodard (2006) stated "accessibility refers to not only architecture but also to the programs provided and availability of information" (p. 6). Furthermore, Yoh, Mohr, and Gordon (2008) found in their study of the satisfaction level of college students with physical disabilities toward the recreation facilities on their campus that use and satisfaction with the recreational sport facilities by these students was significantly low, with over two-thirds of respondents claiming they used the facilities less than five times per semester. The implication of this finding is that while campus recreation administrators and staff may be in compliance with ADA regulations in terms of the accessibility of their facilities, accessibility of programs and information must also be high priorities.

The ADA requires covered entities to make modifications to policies, practices, or procedures when necessary to give individuals with disabilities the opportunity to participate in programs and activities. Many of the institutions reviewed provided either an e-mail or telephone contact to request accommodations or more program information, which was the extent of the information readily available to people with disabilities. Some provided information on disability specific programs. While separate programs may be offered to ensure equal opportunity, an individual with a disability still has the right to choose to participate in the regular program (U.S. Department of Justice, 2002). NIRSA espouses a commitment to equity and inclusion and a core belief that all people should have the opportunity to participate in physical activity and recreation (NIRSA (a), n. d.). Therefore, inclusive campus recreation programs should provide the same choices and opportunities for all participants. Exclusion can occur through negative attitudes and prejudices of staff or other participants, social segregation, structural and communication barriers, and lack of programmatic accommodations.

The fourth and final theme was a direct reflection of the welcoming environment in how well the website communicated accommodations and support services for individuals with disabilities. This theme related to the provision of trained staff and support services specifically for individuals with disabilities. For example, one school in the study provided access to an inclusion specialist and listed a specific description of what this person could do for those with disabilities. While the majority of the institutions' websites reviewed in this study did not convey support services, this was not a new finding as Fujii and Woodard (2006) came to similar conclusions. Furthermore, in a study of NIRSA core competencies by Sturts (2015), campus recreation professionals were asked to rate the importance and their performance in programming efforts for students with disabilities. The result concurred with the findings of current study in that Sturts found providing programs for students with disabilities was the lowest rated in both importance and performance. Respondents rated importance of providing programs for students with disabilities higher than their perceived performance on this subcompetency, yet both were lower in comparison with other subcompetencies under programming. 
While the 14 institutions comprising the Big Ten certainly do not represent all campus recreation programs, using these schools as a sample of major universities and the inclusiveness they provide for students, faculty, and staff with disabilities who may desire to participate in campus recreation programs was extremely revealing. In the last 25 years, the influence of the ADA has leaped many hurdles on behalf of people with disabilities, but it is clear there is still a lot of work yet to accomplish. While some of the institutions in this study were found to be doing a good job of inclusiveness and presenting a welcoming environment, others have much more work to do in creating that type of inclusive culture on their campuses.

Many legal and compliance issues involving students with disabilities may arise from an institution's failure to provide integrated programs and alternative programming required by this cohort of students. In most cases, a one-size-fits-all approach does not adequately meet the requirements set forth by the ADA. As a result, the more specific campus recreation programs can be in identifying and promoting the programs and resources available for students with disabilities and providing a direct contact for more assistance, the more welcoming that program will appear.

\section{Recommendations}

How can campus recreation programs provide the welcoming environment needed to attract, encourage and include participants with disabilities in their programs? The first step is to have an organizational commitment to accessibility and inclusion. That commitment should promote equal opportunity for all participants, integration, independence, respect, and dignity. One of the steps of planning for accessibility and inclusion is to adopt an inclusion statement that reflects the organization has a commitment to accessibility and inclusion. Terms such as "community" which at the surface can appear to provide a sense of inclusion can also be too generic as well. Including references to "inclusion," "integration," "all abilities," and even specifying "people with disabilities" in the statement would directly identify people with disabilities as part of the community. Incorporate the inclusion statement on the campus recreation home webpage and in all publications, promotional and marketing materials. Elevate the accessibility and inclusion information links to the home page of the campus recreation website so they are easy to find. Remember, the farther one has to a navigate levels within a website to obtain information on accessibility and inclusion the less the perception of the commitment.

Students with disabilities may approach information gathering first from the office of disability student services. Campus recreation programs should establish a connection with the office of disability student services and vice versa with each program having a link on its respective website for the other's programs and services. This is a relatively easy task to complete, yet the majority of schools in the study sample failed to do so.

Designate a staff person to be the accessibility/inclusion coordinator for the campus recreation programs. Information about what and how accommodations can be provided and contact information on how to obtain the accommodations should be included on the website and in all promotional materials. The inclusion/ accessibility coordinator should be trained in working with people with disabilities and be able to coordinate and provide training for other staff and volunteers who 
work in the program. Tap into valuable resources for training and support in the academic programs of adapted physical education and recreation therapy if those are available on campus, as well as the disability student services personnel.

Devoting resources needed to achieve accessibility and inclusion must be part of the fabric of conducting the overall program including both staff and financial resources. While the ADA does not require separate adapted recreation programs for people with disabilities, neither does it restrict an entity for providing such programs (U.S. Department of Justice, 2015). Inclusion should be viewed as part of a spectrum of opportunities including full integration without modifications to disability specific and separate programs. The more specific the information regarding programs and activities available for students with disabilities, the more inviting and welcoming the campus program will be perceived.

Adequate training for staff, volunteers, or peers in understanding the needs of and interacting with people with disabilities is critical to overcoming attitudinal barriers and facilitating needed program accommodations. Resources should be available to purchase or rent adapted equipment to enable participation in programs and the availability of that equipment should be advertised and promoted.

Campus recreation programs should market the accessibility of their facilities as evidence of their commitment to providing an inclusive environment. Facilities should be evaluated for accessibility, especially those areas that have been included for the first time in the 2010 ADA Standards (e.g., exercise machines and equipment, golf facilities, sports facilities, saunas and steam rooms, and swimming pools). If noncompliant areas are identified, develop a plan for how and when the barrier will be removed so that resources can be budgeted. New construction and alterations must comply with the 2010 ADA Standards. "An alteration is defined as remodeling, renovating, rehabilitating, reconstructing, changing or rearranging structural parts or elements, ... or making other changes that affect (or could affect) the usability of the facility" (U.S. Department of Justice, 2015, p. 12). Thus, resodding intramural fields would affect the usability of that facility and trigger compliance issues with the 2010 Standards. Sport facilities (e.g., play fields) require an accessible route that connects both sides of courts and playing fields allowing coaches, players, and spectators equal access to seating and to the different view perspectives (York, 2013).

\section{Conclusion}

The ADA has been a law for 25 years providing people with disabilities the right and opportunity to participate in programs throughout American society. Programs and organizations falling under the auspices of Titles II and III of the ADA may technically be in compliance with the law, yet may not be achieving a welcoming environment. Although creating a welcoming environment is not a legal requirement, it is an important characteristic exhibiting best practices of inclusion. In a content analysis of the websites of campus recreation programs at 14 major universities, known as the Big Ten, many could not demonstrate a welcoming environment for people with disabilities via their website. This finding reveals there is still work that needs to be done to meet the full intent of the ADA, or the spirit of the law.

A limitation of this study is the possibility that the campus recreation programs reviewed could actually be much more welcoming and inclusive than their websites 
revealed. Yet, the reality is first impressions are formed for better or for worse when browsing an organization's website. Campus recreation administrators must take this into consideration, specifically in regards to people with disabilities. Further, recommendations for future study in this area are to 1) replicate the study, 2) conduct similar studies in other geographic areas involving a variety of institutions, 3 ) involve more coders to view the website which will help to strengthen the reliability of the content analysis, 4) follow up via phone inquiries about accommodations each program would make for a student with a disability and the ease of which to get this information, and 5) consider nonverbal communication variables such as flow of movement, facility decorations and signage, path of travel options and similar invisible inclusion attributes.

Even in light of the limitations, the results proved disappointing that on face value a more welcoming environment was not found at a majority of the universities involved. The conclusion of this study is that campus recreation programs need to do a better job in implementing the best practice of creating a welcoming environment for students with disabilities. While there are many good steps being taken to demonstrate an inclusive culture, the evidence was overwhelming that more steps must be taken.

\section{References}

Americans with Disabilities Act, 42 U.S. Code $§ 12132$ (1990).

Anderson, L., \& Kress, C.B. (2003). Inclusion: Including people with disabilities in parks and recreation opportunities. State College, PA: Venture Publishing.

Berg, B.L., \& Lune, H. (2012). Qualitative research methods for the social sciences (8th ed.). San Francisco, CA: Peachpit Press.

Dieser, R., \& Scholl, K.G. (2010). Inclusive recreation history and legislation. In H. Kinetics (Ed.), Inclusive recreation: Programs and services for diverse populations. Champaign, IL: Human Kinetics.

Forrester, S. (2015). Benefits of collegiate recreational sports participation: Results from the 2013 NASPA assessment and knowledge consortium study. Recreational Sports Journal, 39(1), 2-15. doi:10.1123/rsj.2015-0005

Fujii, T., \& Woodard, R. (2006). Accessibility in campus recreation programs. Palaestra, 22(3), 6-7.

Gillespie, M. (2002). Attitudes of university students toward an integrated campus recreation program. Palaestra, 18(3), 27-31.

Green, F.P., \& De Coux, V. (1996). Inclusion of students with disabilities in campus recreational sports programs. NIRSA Journal, 20(2), 34-37.

Henderson, K.A., \& Bialeschki, M.D. (2002). Evaluating leisure services making enlightened decisions (2nd ed.). State College, PA: Venture Publishing, Inc.

Hironaka-Juteau, J.H., \& Crawford, T. (2010). Introduction to inclusion. In H. Kinetics (Ed.), Inclusive Recreation: Programs and services for diverse populations. Champaign, IL: Human Kinetics.

Kaltenbaugh, L.P., Molnar, J.C., Bonadio, W.N., Divito, K., \& Roeder, J. (2011). Impact of marketing strategies on campus recreational sports departments. Recreational Sports Journal, 35(2), 86-94.

Kerr-Downs Research. (2003). Value of recreational sports on college campuses: Determinants of college satisfaction and success. Recreational Sports Journal, 27(1), 17-31.

Krippendorff, K. (2013). Content analysis: An introduction to its methodology (3rd ed.). Los Angeles, CA: Sage. 
Landis, J.R., \& Koch, G.G. (1977). The measurement of observer agreement for categorical data. Biometrics, 33, 159-174. doi:10.2307/2529310

Leuchovius, D. (2003). ADA Q \& A...The ADA, Section 504 \& Postsecondary Education. Pacer Center Action Information Sheets, PHP-c51g.

Mull, R.F., Forrester, S.A., \& Barnes, M.L. (2013). Recreational sport programming (5th ed.). Urbana, IL: Sagamore Publishing.

National Center on Domestic Violence, Trauma \& Mental Health (2011). Tips for creating a welcoming environment. Retrieved from http://nationalcenterdvtraumamh.org/wp-content/uploads/2012/01/Tipsheet_Welcoming-Environment_NCDVTMH_Aug2011.pdf.

National Center on Health. Physical Activity and Disability (n.d.). The pathway to inclusion: From principle to profit. Retrieved from http://www.nchpad.org/602/2551/2008-12 Iss ue The Pathway to Inclusion From Principle to Profit.

National Intramural-Recreational Sports Association. (a) (n.d.) Core Beliefs. Retrieved from http://nirsa.net/nirsa/about/strategic-plan/

Neuman, W.L. (2004). Basics of social research qualitative and quantitative approaches. Boston, MA: Pearson Education Inc.

NIRSA/NASPA. (2010). NIRSA/NASPA consortium campus recreation impact study. Retrieved from http://www.nirsa.org/docs/Discover/Research/Campus_Rec_Impact_ Study.PDF

Ohio State University Recreational Sports. (2015). Recreational sports historical timeline. Retrieved from http://www.recsports.osu.edu/who-we-are/rec-sports-historical-timeline

Pelka, F. (2012). What have we done: An oral history of the disability rights movement. Amherst and Boston, MA: University of Massachusetts Press.

Potter, J.W. (1996). An analysis of thinking and research about qualitative methods. Mahwah, NJ: Erlbaum.

Ross, C.M., \& Phillips, R. (1995). The Americans with Disabilities Act (ADA) and its impact on recreational sports facilities. NIRSA Journal, 19(3), 38-43.

Roth, D., \& Hudson, S.D. (1994). The impact of the Americans with Disabilities Act on recreational sports programs. NIRSA Journal, 18(3), 22-25.

Stroman, D. (2003). The disability rights movement: From deinstitutionalization to selfdetermination. Lanham, MD: University Press of America.

Sturts, J.R. (2015). Perceived importance and performance of NIRSA core competencies by professional in collegiate recreational sports. (Unpublished doctoral dissertation). Indiana University: Bloomington, IN.

University of Michigan. (2015). 100 years of recreational sports. Introduction to the AA http://recsports.umich.edu/timeline/874/100-years-recreational-sports

United States Access Board. (a). About the Architectural Barriers Act and Other Disability Laws. [Online]. Available at: http://www.access-board.gov/about/aboutABA/brochure. htm.

United States Access Board. (b). Americans with Disabilities Act (ADA) of 1990, as amended. [Online]. Available at: http://www.access-board.gov/about/laws/ADA.htm

United States Department of Education. (2013). Dear colleague letter, January 25, 2013. Retrieved from http://www2.ed.gov/about/offices/list/ocr/letters/colleague-201301-504. html

United States Department of Justice. (a) (n.d.). 1991 ADA Standards for Accessible Design. Retrieved from http://www.ada.gov/1991ADAstandards_index.htm.

United States Department of Justice. (b) (n.d.). Introduction to the ADA. Retrieved from http://www.ada.gov/ada_intro.htm

United States Department of Justice. (2002). Title II highlights. Retrieved from http://www. ada.gov/t2hlt $95 . h t m$

United States Department of Justice. (2010). Fact sheet: Adoption of the 2010 Standards for Accessible Design. Retrieved from http://www.ada.gov/regs2010/factsheets/2010_Standards_factsheet.html 
United States Department of Justice. (2015). ADA update: A primer for state and local governments. Retrieved from http://www.ada.gov/regs2010/titleII_2010/titleII_primer.pdf

Weber, R.P. (1990). Basic content analysis (2nd ed.). Newbury Park, CA: Sage Publications, Inc.

White, M.D., \& Marsh, E.E. (2006). Content analysis: A flexible methodology. Library Trends, 55(1), 22-45. doi:10.1353/lib.2006.0053

Yoh, T., Mohr, M., \& Gordon, B. (2008). Assessing satisfaction with campus recreation facilities among college students with physical disabilities. Recreational Sports Journal, 32, 106-113.

York, S.L. (2013). Design of accessible and usable recreation environments. In D.R. Austin \& Y. Lee (Eds.), Inclusive \& special recreation: Opportunities for persons from diverse populations to flourish (6th ed., pp. 201-228). Urbana, IL: Sagamore Publishing. 